\title{
Disponibilidade domiciliar de lipídeos para consumo e sua relação com os lipídeos séricos de adolescentes
}

\author{
Household availability of lipids for consumption and its relationship with serum lipids in adolescents
}

\author{
Vivian Siqueira S. Gonçalves', Otaviana Cardoso Chaves², Sônia Machado R. Ribeiro ${ }^{3}$ Luciana Ferreira R. Sant'Ana ${ }^{4}$, Sylvia do \\ Carmo C. Franceschini ${ }^{5}$, Silvia Eloiza Priore ${ }^{6}$
}

\section{RESUMO}

Objetivo: Relacionar a disponibilidade domiciliar de lipídeos para consumo e o perfil lipídico sérico de adolescentes.

Métodos: Estudo transversal observacional. Avaliaram-se 95 adolescentes púberes de 10 a 13 anos de escolas públicas em Viçosa, Minas Gerais. Utilizou-se uma lista de compras mensal para avaliar a disponibilidade de alimentos per capita. Coletou-se amostra de sangue dos adolescentes, adotando-se o preconizado pela Sociedade Brasileira de Cardiologia como referência para adequação do perfil lipídico. Para as variáveis categóricas realizou-se o teste do qui-quadrado e calculou-se a Odds Ratio. Para comparar os grupos, aplicaram-se os testes Mann-Whitney ou $t$ de Student, Kruskall-Wallis ou ANOVA. Verificou-se relação entre exames bioquímicos e disponibilidade de lipídeos pelas correlações de Spearmam ou de Pearson.

Resultados: Da energia total disponível, 27,2\% foram provenientes de lipídeos. A disponibilidade de ácidos graxos saturados, monoinsaturados e poli-insaturados esteve de acordo com a recomendação de consumo. Em relação aos lipídeos séricos, 51,9\% dos meninos e 55,8\% das meninas apresentaram alterações de colesterol total $(p=0,66) ; 18,6$ e 25,6\%, dos meninos e das meninas, respectivamente, apresentaram elevação de triglicerídeos, (meninas > meninos; $p=0,02)$. Em relação ao LDL, 21,2 e 32,6\% dos meninos e das meninas, respectivamente, apresentaram alterações $(p=0,91)$. A prevalência de inadequação para HDL foi de 26,9 e 30,2\% $(p=0,93)$, para meninos e meninas, respectivamente. Não houve correlação entre os lipídeos disponíveis para consumo e os lipídeos séricos. Os adolescentes que possuíam mais que $35 \%$ de lipídeos disponíveis para consumo tiveram 9,1 vezes mais chances de apresentarem alterações de colesterol total (IC95\% 1,81-61,74).

Conclusões: É possível que adolescentes com maior disponibilidade de lipídeos para consumo apresentem colesterol total mais elevado.

Palavras-chave: gorduras na dieta; dislipidemias; nutrição do adolescente.

\section{ABSTRACT}

Objective: To analyze the correlation between household availability of lipids to be consumed and the serum lipid profile in adolescents.

Methods: This cross sectional study enrolled 95 adolescents aged 10-13 years, selected by random raffle in public schools of Viçosa, Southeast Brazil. A monthly shopping
Instituição: Universidade Federal de Viçosa (UFV), Viçosa, MG, Brasil ${ }^{1}$ Graduanda em Nutrição na UFV, Viçosa, MG, Brasil

${ }^{2}$ Mestre em Ciência da Nutrição pela UFV; Professora Substituta do Departamento de Nutrição da Universidade Federal dos Vales do Jequitinhonha e Mucuri (UFVJM), Diamantina, MG, Brasil

${ }^{3}$ Doutora em Bioquímica Agrícola pela UFV; Professora do Departamento de Nutrição e Saúde da UFV, Viçosa, MG, Brasil

${ }^{4}$ Doutora em Ciência e Tecnologia de Alimentos pela UFV; Professora do Departamento de Nutrição e Saúde da UFV, Viçosa, MG, Brasil ${ }^{5}$ Doutora em Ciências pela Universidade Federal de São Paulo (Unifesp); Professora do Departamento de Nutrição e Saúde da UFV, Viçosa, MG, Brasil ${ }^{6}$ Doutora em Nutrição pela Unifesp; Professora do Departamento de Nutrição e Saúde da UFV, Viçosa, MG, Brasil
Endereço para correspondência:

Vivian Siqueira S. Gonçalves

Travessa Felício Brandi, 40/604 - Centro

CEP 36570-000 - Viçosa/MG

E-mail: vivian.goncalves@ufv.br

Conflito de interesse: nada a declarar

Recebido em: 5/6/2011

Aprovado em: 31/10/2011 
list was used to evaluate the availability of food per capita. A blood sample was collected from each adolescent and Brazilian Cardiology Society guideline was adopted as a reference for lipid profile adequacy. For categorical variables, the chisquare was applied and the Odds Ratio calculated. For comparison between groups, Mann-Whitney or Student's $t$ test, Kruskall-Wallis test or ANOVA were applied. Association between biochemical parameters and lipid availability was evaluated by Spearman or Pearson correlation tests.

Results: $27.2 \%$ of the total available energy came from lipids. The availability of saturated fatty acids, monounsaturated and polyunsaturated agreed with the recommendation for their consumption. Regarding serum lipids, $51.9 \%$ of boys and $55.8 \%$ of girls showed alteration in total cholesterol $(p=0.66) ; 18.6$ and $25.6 \%$ of boys and girls, respectively, showed increased triglycerides level (girls $>$ boys; $p=0.02$ ). In relation to LDL, 21.2 and $32.6 \%$ of boys and girls, respectively, showed alteration $(p=0.91)$. The prevalence of inadequacy for HDL was of 26.9 and $30.2 \%$ of boys and girls, respectively $(p=0.93)$. There was no correlation between the available lipids for consumption and the serum lipids. The teenagers with more than $35 \%$ of lipids to be consumed had 9.1-fold higher levels of total cholesterol (95\% CI 1.81-61.74).

Conclusions: It is possible that adolescents with higher lipid availability at home show higher total cholesterol.

Key-words: dietary fats; dyslipidemias; adolescent nutrition.

\section{Introdução}

$\mathrm{Na}$ adolescência, definida pela Organização Mundial de Saúde (OMS) como o período compreendido entre dez e 19 anos, verificam-se mudanças fisiológicas, psicológicas e sociais que podem ocorrer de forma variada entre os indivíduos. Trata-se de um período de vulnerabilidade nutricional e consolidação de hábitos alimentares que, não sendo adequados, podem trazer o risco de doenças ${ }^{(1)}$.

A Pesquisa de Orçamento Familiar (POF), realizada nas áreas metropolitanas do Brasil entre os anos 2002 e 2003, mostrou que a dieta da população brasileira caracterizava-se pelo aumento do teor de lipídeos em geral e de gordura saturada, apontando ainda que a evolução dos padrões de consumo alimentar nas últimas três décadas tendeu ao aumento de fatores de risco para o desenvolvimento da obesidade, diabetes, doenças cardiovasculares, certos tipos de câncer e outras enfermidades crônicas( ${ }^{(2)}$. A POF 2008-2009 apontou que o excesso de peso na população vem aumentando desde meados da década de 1970 e é encontrado em cerca de metade dos brasileiros ${ }^{(3)}$. Campos $e t a^{(4)}$ ressaltam que, independentemente da origem étnica, existem evidências de que indivíduos que consomem quantidades elevadas de lipídeos, principalmente do tipo saturado, geralmente têm níveis elevados de colesterol sérico e maior risco de desenvolverem doenças cardiovasculares.

Os lipídeos são macronutrientes que desempenham funções energéticas, estruturais e hormonais no organismo, conferindo sabor aos alimentos e sensação de saciedade, além de veicular as vitaminas lipossolúveis, sendo quase todos compostos por ácidos graxos. Os ácidos graxos saturados são encontrados principalmente em gorduras animais. Os ácidos graxos insaturados se dividem em monoinsaturados, presentes em óleos como o de oliva, e em poli-insaturados, comuns em óleos de soja, canola e peixes, englobando também os chamados trans, presentes em alguns produtos industrializados. Existem ácidos graxos poli-insaturados que não podem ser sintetizados pelo organismo humano, denominados essenciais, que devem ser fornecidos pela alimentação, dentre eles destacam-se os ômega 3 e $6^{(5)}$.

O perfil lipídico é definido pelas determinações bioquímicas do colesterol total (CT), high-density lipoprotein (HDL), low-density lipoprotein (LDL) e triglicerídeos (TG) após jejum de 12 a 14 horas $^{(6)}$, podendo ser utilizado para avaliar o balanço adequado de lipídeos sanguíneos.

Nesse contexto, torna-se importante verificar a disponibilidade de alimentos nos domicílios de adolescentes, pois o consumo exagerado de lipídeos pode contribuir com um padrão alimentar inadequado, capaz de acarretar consequências negativas sobre sua saúde futura. Diante disso, este estudo teve como objetivo verificar a existência de relação entre a disponibilidade domiciliar de lipídeos para consumo e o perfil lipídico de adolescentes residentes em Viçosa, Minas Gerais.

\section{Método}

Tratou-se de um estudo transversal e observacional, tendo como unidade o indivíduo. A seleção da amostra foi baseada em informações do Instituto Nacional de Estudos e Pesquisas Educacionais Anísio Teixeira (INEP), que apontou, em 2008, a presença de 25 escolas oferecendo ensino fundamental no município de Viçosa ${ }^{(7)}$. Trabalhou-se com $100 \%(n=19)$ das escolas públicas da zona urbana. 
O estudo, integrante de uma pesquisa de maior abrangência ${ }^{(8)}$, utilizou para o cálculo amostral o número de adolescentes total entre a faixa etária de dez a 13 anos do município, que segundo o Datasus ${ }^{(9)}$ era de 4.815, a frequência de excesso de peso de $10 \%$, obtida em estudos anteriores com a população local de adolescentes ${ }^{(10)}$, com margem aceitável de variação de $2 \%$ e nível de confiança de $99 \%$, totalizando 92 adolescentes a serem estudados. Devido à possível perda amostral esperada e para maior homogeneidade e distribuição dos dados em relação ao gênero e faixa etária, acrescentaram-se $20 \%$ ao cálculo inicial, totalizando 110 adolescentes.

As escolas foram visitadas e todos os alunos que demonstraram interesse em participar foram submetidos a uma seleção aleatória. A presença de doenças crônicas e/ou o uso de medicamentos que alterassem o metabolismo lipídico constituíram critérios de exclusão. Ao final, foram sorteados 146 adolescentes, de ambos os gêneros, que compareceram para a avaliação da maturação sexual, sendo 25 excluídos por serem pré-puberes e um por desistência. Assim, 120 adolescentes realizaram os exames bioquímicos, sendo possível obter a lista de compras de alimentos mensal da família com dados relacionados aos gêneros alimentícios e quantidades de 95 adolescentes, o que constituiu o número final da amostra. Todos os adolescentes residiam com suas famílias em Viçosa. Eles foram considerados púberes, segundo classificação de Tanner ${ }^{(11)}$, após serem avaliados por um médico pediatra.

A lista de compras da família foi utilizada para avaliar a disponibilidade de alimentos per capita nos domicílios dos adolescentes e continha informações sobre a quantidade mensal de gêneros alimentícios adquiridos. Tais informações foram obtidas por meio de cupons fiscais ou pela estimativa da quantidade e frequência de aquisições referentes a um único mês feita pelos responsáveis ${ }^{(2,12)}$. Para o cálculo estimado de alimentos disponíveis para consumo per capita diário dividiu-se a quantidade mensal de alimentos disponíveis no domicílio pelo número de moradores e pelo número de dias do referido mês ${ }^{(12)}$. Os alimentos que não tiveram especificada a quantidade foram desconsiderados.

A composição dos alimentos e os teores de lipídeos constituintes dos mesmos foram estabelecidos pelo software Diet Pro $^{\circledR}$ 4.0, a partir da tabela de dados dietéticos americana (USDA). Para os alimentos que não faziam parte da tabela, foram utilizados seus rótulos ou a tabela de composição química de alimentos ${ }^{(13)}$.
Os valores de lipídeos da dieta foram comparados com o preconizado pelo Institute of Medicine (IOM) ${ }^{(14)}$, utilizando a Acceptable Macronutrient Distribution Range (AMDR). Segundo o IOM, 25 a $35 \%$ da ingestão energética total devem ser proveniente de lipídeos, sendo 5 a 10\% oriundos de ômega-6 (AGP $\omega-6$ ) e 0,6 a 1,2\% de ômega-3 (AGP $\omega-3$ ).

O percentual de gordura saturada (AGS) foi considerado elevado quando ultrapassou $7 \%$ e de monoinsaturada (AGM) quando esteve acima dos $20 \%$ do total calórico diário ${ }^{(15)}$. Mensurou-se a disponibilidade de óleo de soja, muito utilizado pela população brasileira, sendo considerada adequada a quantidade per capita estipulada pela pirâmide alimentar adaptada à população brasileira, $16 \mathrm{~mL}^{(16)}$.

A contribuição das carnes diversas para a composição lipídica final das listas de compras também foi considerada e integrou os dados apresentados, uma vez que $100 \%$ das famílias declararam adquirir este alimento regularmente.

A coleta de sangue para determinar o perfil lipídico sérico foi realizada por profissionais tecnicamente capacitados do Laboratório de Análises Clínicas da Divisão de Saúde da Universidade Federal de Viçosa, após jejum de 12 horas, com todo material descartável. Foram coletados $10 \mathrm{~mL}$ de sangue venoso, o material foi centrifugado em centrífuga Excelsa (modelo $206 \mathrm{BL}$ ) por 10 minutos a $3.500 \mathrm{rpm}$ logo após a coleta (observando tempo suficiente para a coagulação do sangue). O CT, HDL e TG foram avaliados por método enzimático, com automação pelo equipamento Cobas Mira Plus (Roche ${ }^{\circledR}$ ); o LDL foi calculado a partir da fórmula de Friedwald ${ }^{(17)}$. Os resultados foram avaliados segundo a I Diretriz de Prevenção da Aterosclerose na Infância e na Adolescência ${ }^{(15)}$.

Para análise das variáveis categóricas realizou-se o teste do qui-quadrado e calculou-se a Odds Ratio (OR). As variáveis quantitativas foram testadas quanto à normalidade pelo teste de Kolmogorov-Smirnov, a partir da qual se selecionou o melhor teste para comparar dois grupos (Mann-Whitney ou $t$ de Student) ou três grupos (KruskallWallis ou ANOVA). A relação entre exames bioquímicos e a disponibilidade de lipídeos foi verificada pelo teste de correlação de Spearmam ou Pearson. Foram utilizados os softwares Sigma Statistic ${ }^{\circledast} 2.0$ e Epi-Info 6.0, sendo significante $p<0,05$.

Os participantes apresentaram termo de consentimento livre e esclarecido assinado pelos pais ou responsáveis. $\mathrm{O}$ estudo foi aprovado pelo Comitê de Ética em Pesquisa com Seres Humanos da Universidade Federal de Viçosa. 


\section{Resultados}

Os meninos totalizaram $54,7 \%(\mathrm{n}=52)$ da amostra. A energia diária total disponível para ambos os gêneros apresentou mediana de $1.952 \mathrm{kcal}(862-4.263)$; para o grupo dos meninos $1.908 \mathrm{kcal}(862-4.263)$ e $1.959 \mathrm{kcal}$ (935-3.864) para o grupo das meninas $(p=0,70)$.

A Tabela 1 traz a disponibilidade calórica per capita proveniente de lipídeos de adição e constituição dos

Tabela 1 - Disponibilidade calórica per capita diária proveniente de lipídeos, por gênero

\begin{tabular}{lccc}
\hline & $\mathbf{n}$ & $\overline{\mathbf{X}} \pm$ DP & Md (mín-máx) \\
\hline Masculino & 52 & $592,8 \pm 263,2$ & $532,9(145,4-1.495,8)$ \\
Feminino & 43 & $591,4 \pm 261,9$ & $528,9(235,6-1.510,3)$ \\
Total & 95 & $592,2 \pm 261,3$ & $530,0(145,4-1.510,3)$ \\
\hline
\end{tabular}

$\mathrm{X} \pm \mathrm{DP}$ : média e desvio padrão; Md: mediana; mín-máx: valores mínimo e máximo; Teste de Mann-Whitney $(p=0,87)$

Tabela 2 - Adequação da disponibilidade per capita de lipídeos obtidos pela lista de compras da família de adolescentes entre dez e 13 anos, por gênero

\begin{tabular}{lccc}
\hline & $\begin{array}{c}\text { Acima } \\
(>35 \%)\end{array}$ & $\begin{array}{c}\text { Adequado } \\
(\mathbf{2 5 - 3 5 \% )}\end{array}$ & $\begin{array}{c}\text { Abaixo } \\
(<25 \%)\end{array}$ \\
\hline & $\%$ & $\%$ & $\%$ \\
Masculino $(\mathrm{n}=52)$ & 21,2 & 55,8 & 23,1 \\
Feminino $(\mathrm{n}=43)$ & 16,3 & 58,2 & 25,6 \\
Total & 18,9 & 56,8 & 24,2 \\
\hline
\end{tabular}

Teste do qui-quadrado: Acima versus Adequado $(p=0,58)$; Adequado versus Abaixo $(p=0,90)$; Acima versus Abaixo $(p=0,56)$ alimentos. Não houve diferença entre os gêneros para esse parâmetro $(p=0,87)$. Em relação à energia total, a disponibilidade foi de $27,7 \%$ para os meninos e $27,0 \%$ para as meninas. Para a amostra total houve disponibilidade de $27,2 \%$.

$\mathrm{Na}$ Tabela 2 encontra-se a adequação do percentual de lipídeos da dieta, de acordo com o recomendado. Não houve diferença, entre os gêneros.

Todas as famílias entrevistadas adquiriram óleo de soja. A análise quantitativa desse produto apontou 23 (5-72mL) per capita disponíveis diariamente, o que significou aproximadamente $760 \mathrm{~mL}$ de óleo de soja mensais disponíveis para cada membro da família.

O tipo de lipídeo, como fonte das classes de ácidos graxos, está disponível na Tabela 3. Na Tabela 4, apresentam-se os valores médios e medianos do perfil lipídico sérico dos adolescentes. Em relação à prevalência de alterações, 69,8\% (n=67) apresentaram pelo menos um e $5,3 \%(\mathrm{n}=5)$ apresentaram os quatro exames bioquímicos alterados. Verificou-se que 51,9\% dos meninos e 55,8\% das meninas tiveram alterações nos valores de CT; 18,6 e $25,6 \%$ dos meninos e das meninas, respectivamente, apresentaram elevação de TG. Em relação ao LDL, 21,1 e $32,6 \%$ de meninos e meninas possuíam valores elevados para esse parâmetro, respectivamente. A prevalência de inadequação para HDL foi de 26,9 e $30,2 \%$, respectivamente, para meninos e meninas.

Na Tabela 5, encontram-se as correlações entre o perfil lipídico sérico e a disponibilidade de lipídeos para consumo dos adolescentes avaliados.

Tabela 3 - Quantidade e classe de ácidos graxos disponíveis para consumo pelos adolescentes de dez a 13 anos, por gênero

\begin{tabular}{|c|c|c|c|c|c|c|c|c|c|c|}
\hline \multirow[b]{2}{*}{$\begin{array}{l}\text { Lipídeos } \\
\text { (g) }\end{array}$} & \multicolumn{3}{|c|}{ Total $(n=95)$} & \multicolumn{3}{|c|}{ Masculino $(n=52)$} & \multicolumn{3}{|c|}{ Feminino $(n=43)$} & \multirow[b]{2}{*}{$\begin{array}{c}\text { Valor } \\
p\end{array}$} \\
\hline & $\overline{\mathbf{X}}_{ \pm} \mathrm{DP}$ & $\begin{array}{c}\text { Md } \\
\text { (mín-máx) }\end{array}$ & $\%$ & $\overline{\mathrm{X}} \pm \mathrm{DP}$ & $\begin{array}{c}\text { Md } \\
\text { (mín-máx) }\end{array}$ & $\%$ & $\overline{\mathbf{X}} \pm \mathrm{DP}$ & $\begin{array}{c}\text { Md } \\
\text { (mín-máx) }\end{array}$ & $\%$ & \\
\hline LT & $65,8 \pm 29,0$ & $\begin{array}{c}58,8 \\
(16,2-167,8)\end{array}$ & 27,2 & $66,5 \pm 28,9$ & $\begin{array}{c}59,2 \\
(16,2-166,2)\end{array}$ & 27,7 & $64,9 \pm 29,5$ & $\begin{array}{c}58,7 \\
(16,2-167,8)\end{array}$ & 27,0 & 0,76 \\
\hline AGS & $16,5 \pm 8,8$ & $\begin{array}{c}14,6 \\
(3,0-49,9)\end{array}$ & 6,7 & $16,3 \pm 8,5$ & $\begin{array}{c}14,7 \\
(3,0-43,6)\end{array}$ & 6,9 & $16,7 \pm 9,3$ & $\begin{array}{c}14,5 \\
(3,0-49,9)\end{array}$ & 6,7 & 0,99 \\
\hline AGM & $19,5 \pm 10,4$ & $\begin{array}{c}17,1 \\
(4,3-67,5)\end{array}$ & 7,9 & $19,5 \pm 9,8$ & $\begin{array}{c}17,4 \\
(4,3-54,9)\end{array}$ & 8,2 & $19,6 \pm 11,2$ & $\begin{array}{c}17,0 \\
(4,3-67,5)\end{array}$ & 7,8 & 0,78 \\
\hline $\begin{array}{l}\text { AGP } \\
\omega-6\end{array}$ & $17,4 \pm 8,1$ & $\begin{array}{c}16,3 \\
(1,2-45,5)\end{array}$ & 7,5 & $17,9 \pm 9,0$ & $\begin{array}{c}16,8 \\
(1,2-45,5)\end{array}$ & 7,9 & $16,9 \pm 7,1$ & $\begin{array}{c}15,9 \\
(5,8-32,5)\end{array}$ & 7,3 & 0,53 \\
\hline $\begin{array}{l}\text { AGP } \\
\omega-3\end{array}$ & $2,3 \pm 1,1$ & $\begin{array}{c}2,1 \\
(0,5-5,8)\end{array}$ & 0,9 & $2,3 \pm 1,2$ & $\begin{array}{c}2,2 \\
(0,5-5,8)\end{array}$ & 1,1 & $2,2 \pm 0,9$ & $\begin{array}{c}2,0 \\
(0,6-4,3)\end{array}$ & 0,9 & 0,54 \\
\hline
\end{tabular}

$\mathrm{X} \pm \mathrm{DP}$ : média e desvio padrão; Md: mediana; mín-máx: valores mínimo e máximo; \%: percentual referente ao total de calorias; LT: lipídeos totais; AGS: ácidos graxos saturados; AGM: ácidos graxos monoinsaturados; AGP $\omega$-3: ácidos graxos poli-insaturados ômega-3; AGP $\omega$-6: ácidos graxos poli-insaturados ômega- 6 
Os resultados da comparação entre o perfil lipídico sérico, de acordo com a adequação da disponibilidade lipídica para consumo em seus domicílios, encontram-se na Tabela 6.

Através da OR, verificou-se que os adolescentes que possuíam mais que $35 \%$ de lipídeos disponíveis para consumo tinham chances 9,11 vezes maiores (IC95\% 1,81-61,74) de apresentarem alterações de colesterol total que os adolescentes com disponibilidade adequada ou baixa.

\section{Discussão}

Observaram-se alterações importantes no perfil lipídico sérico dos avaliados, notando-se número elevado de famílias com disponibilidade de lipídeos acima de 35\% das calorias totais. Tais fatos podem ser resultado da tendência atual de consumo maior de alimentos energéticos e com alto teor de gorduras.

De acordo com a $\mathrm{POF}^{(2)}$, em 2002 e 2003 no Brasil, a disponibilidade energética média per capita era de $1.800 \mathrm{kcal} / \mathrm{dia}$. Em

Tabela 4 - Perfil lipídico sérico de adolescentes de dez a 13 anos, por gênero

\begin{tabular}{|c|c|c|c|}
\hline & Masculino $(n=52)$ & Feminino $(n=43)$ & \\
\hline Lipídeos (mg/dL) & $\begin{array}{c}\overline{\mathbf{X}} \pm \mathrm{DP} \\
\text { Md (mín-máx) }\end{array}$ & $\begin{array}{c}\overline{\bar{X}_{ \pm} \pm \mathrm{DP}} \\
\text { Md (mín-máx) }\end{array}$ & Valor $p$ \\
\hline CT & $\begin{array}{c}154,9 \pm 25,9 \\
151,5(114,0-259,0)\end{array}$ & $\begin{array}{c}157,5 \pm 30,9 \\
154,0(93,0-221,0)\end{array}$ & 0,65 \\
\hline TG & $\begin{array}{c}68,9 \pm 47,0 \\
55,0(21,0-276,0)\end{array}$ & $\begin{array}{c}81,6 \pm 40,1 \\
72,0(30,0-195,0)\end{array}$ & 0,02 \\
\hline LDL & $\begin{array}{c}88,6 \pm 22,5 \\
86,4(47,0-181,0)\end{array}$ & $\begin{array}{c}88,5 \pm 25,9 \\
82,6(39,8-147,6)\end{array}$ & 0,90 \\
\hline HDL & $\begin{array}{c}52,5 \pm 11,8 \\
49,0(31,0-85,0)\end{array}$ & $\begin{array}{c}52,7 \pm 16,6 \\
52,0(24,0-115,0)\end{array}$ & 0,93 \\
\hline
\end{tabular}

$\mathrm{X} \pm \mathrm{DP}$ : média e desvio padrão; Md: mediana; mín-máx: valores mínimo e máximo; CT: colesterol total; TG: triglicerídeos: LDL: low density lipoprotein; HDL: high density lipoprotein

Tabela 5 - Correlação entre o perfil lipídico e a disponibilidade de lipídeos para consumo por adolescentes de dez a 13 anos

\begin{tabular}{lccccc}
\hline \multirow{2}{*}{ Lipídeos séricos } & \multicolumn{5}{c}{ Lipídeos disponíveis para consumo } \\
\cline { 2 - 6 } & LT & AGS & AGP $\omega-3$ & AGP $\omega-6$ & AGM \\
\hline CT & $-0,2$ & 0,1 & $-0,1$ & $-0,2$ & 0,1 \\
TG & 0,1 & 0,1 & 0,1 & 0,1 & 0,3 \\
LDL & $-0,2$ & 0,1 & $-0,1$ & $-0,2^{*}$ & 0,1 \\
HDL & $-0,1$ & $0,2^{*}$ & $-0,1$ & $-0,1$ & 0,2 \\
\hline
\end{tabular}

LT: lipídeos totais; AGS: ácidos graxos saturados; AGM: ácidos graxos monoinsaturados; AGP $\omega$-3: ácidos graxos poli-insaturados ômega-3; AGP $\omega$-6: ácidos graxos poli-insaturados ômega-6; CT: colesterol total; TG: triglicerídeos; LDL: low density lipoprotein; HDL: high density lipoprotein. " $p<0,05$ (Correlação de Pearson ou Spearman)

Tabela 6 - Perfil lipídico sérico de adolescentes de dez a 13 anos, de acordo com a adequação da disponibilidade de lipídeos para consumo

\begin{tabular}{|c|c|c|c|c|}
\hline & Abaixo (<25\%) & Adequada (25-35\%) & Acima (>35\%) & \\
\hline (mg/dL) & 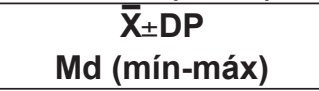 & $\begin{array}{c}\overline{\mathrm{X}}_{ \pm \mathrm{DP}} \\
\text { Md (mín-máx) }\end{array}$ & $\begin{array}{c}\bar{X}_{ \pm D P} \\
\text { Md (mín-máx) }\end{array}$ & Valor $p$ \\
\hline CT & $\begin{array}{c}145,8 \pm 28,3 \\
142,0(93,0-205,0)^{*}\end{array}$ & $\begin{array}{c}156,5 \pm 29,5 \\
152,5(108,0-259,0)\end{array}$ & $\begin{array}{c}167,7 \pm 18,2 \\
164,0(141,0-214,0)^{*}\end{array}$ & 0,01 \\
\hline TG & $\begin{array}{c}72,8 \pm 47,1 \\
64,0(25,0-228,0)^{\text {*\# }}\end{array}$ & $\begin{array}{c}76,9 \pm 45,8 \\
66,5(21,0-276,0)^{\#}\end{array}$ & $\begin{array}{c}70,4 \pm 36,9 \\
65,0(27,0-164,0)^{\star}\end{array}$ & $<0,001$ \\
\hline LDL & $\begin{array}{c}82,5 \pm 23,3 \\
76,4(47,0-130,6)\end{array}$ & $\begin{array}{c}89,1 \pm 26,2 \\
83,9(39,8-181,0)\end{array}$ & $\begin{array}{c}94,7 \pm 15,7 \\
90,3(77,8-145,8)\end{array}$ & 0,06 \\
\hline HDL & $\begin{array}{c}48,8 \pm 10,7 \\
48,0(26,0-75,0) \\
\end{array}$ & $\begin{array}{c}52,1 \pm 14,9 \\
48,5(24,0-115,0) \\
\end{array}$ & $\begin{array}{c}58,9 \pm 13,7 \\
57,5(35,0-82,0) \\
\end{array}$ & 0,06 \\
\hline
\end{tabular}

X $\pm D P$ : média e desvio padrão; Md: mediana; mín-máx: valores mínimo e máximo;CT: colesterol total; TG: triglicerídeos; LDL: low density lipoprotein; HDL: high density lipoprotein. *\#Teste de comparações múltiplas de Dunn: símbolos iguais na mesma linha indicam diferença estatística ( $p<0,05)$ 
2006, utilizando a lista de compras, Barbosa et al ${ }^{(12)}$ estudaram adolescentes do gênero feminino e encontraram disponibilidade diária mediana de $1.868 \mathrm{kcal}$. Comparando-se ao presente estudo, verifica-se o aumento de $7,7 \%$ em relação à $\mathrm{POF}^{(2)}$ e $4,3 \%$ em relação ao trabalho de Barbosa et al ${ }^{(12)}$. A POF 2008-2009(3) aponta maior satisfação da população brasileira com a quantidade de alimentos consumidos: $64,5 \%$ das famílias afirmam ter alimentos em quantidade sempre suficiente em seus lares, percentual maior do que o apresentado em 2002-2003 (53,0\%). Tais fatos podem evidenciar que a população está tendo acesso à maior quantidade de alimentos, o que pode justificar a crescente disponibilidade calórica encontrada.

Entretanto, percentual considerável dos adolescentes avaliados dispunha em seus domicílios de quantidade de lipídeo superior à recomendação para consumo. Esse fato merece atenção, pois se espera que, à medida em que a disponibilidade seja maior, o consumo também possa aumentar. A prevalência de excesso no consumo desse nutriente já é relatada em alguns estudos ${ }^{(18-20)}$. Caso o acesso crescente a alimentos no Brasil não seja acompanhado por estratégias concretas de educação nutricional, as inadequações alimentares provavelmente tenderão a crescer, trazendo consigo o risco de desenvolvimento de doenças crônicas não transmissíveis em faixas etárias cada vez mais precoces.

Os percentuais de disponibilidade de AGS, AGM, AGP $\omega-3$ e $\omega-6$ apresentaram-se dentro das faixas preconizadas pela AMDR, o que significa que os adolescentes dispunham de quantidades e qualidade de lipídeos para que pudessem equilibrar suas refeições. Ressalta-se, no entanto, uma razão de aproximadamente 8:1 dos AGP $\omega$-6 e $\omega$-3. Em estudo de revisão, Barbosa et a ${ }^{(21)}$ abordam as recomendações controversas dessa razão, salientando que, sendo menor, poderia trazer efeitos sinérgicos entre o consumo dos dois ácidos graxos.

Em um estudo realizado com adolescentes do gênero feminino, na faixa etária de 14 a 17 anos $^{(10)}$, houve disponibilidade mediana per capita diária de $24 \mathrm{~mL}$ de óleo de soja; já os valores encontrados no presente estudo são inferiores, mas ambos estão acima do preconizado pela pirâmide alimentar adaptada à população brasileira ${ }^{(16)}$. Este fato pode colaborar para o desequilíbrio da proporção adequada de AGP $\omega-3$ e $\omega-6$, uma vez que se trata de um alimento fonte de AGP $\omega-6^{(21)}$.

As inadequações encontradas para as frações do colesterol sérico (LDL e HDL) merecem destaque, assim como para TG. Diferentes trabalhos com adolescentes apresentaram médias semelhantes às observadas neste estudo $^{(22-25)}$. Em estudo realizado no nordeste brasileiro com adolescentes provenientes de escolas públicas e na mesma faixa etária, Pereira et al ${ }^{(26)}$ encontraram percentual de inadequação para CT de $36,2 \%$, valor abaixo do verificado para meninos e meninas $(51,9$ e $55,8 \%$, respectivamente) neste trabalho. Os autores ainda verificaram, assim como neste estudo, que foram grandes as inadequações nas concentrações de $\operatorname{HDL}(56,0 \%)$, LDL $(14,5 \%)$ e TG $(35,3 \%)$. No México, em um estudo realizado com adolescentes de ambos os gêneros, também foi detectado percentual considerável de alterações no perfil lipídico, sendo $20 \%$ para TG, $20 \%$ para CT, $30 \%$ para LDL e $66 \%$ para $\mathrm{HDL}^{(27)}$.

O comportamento alimentar inadequado, assim como o declínio da atividade física cada vez mais eminente na vida moderna, podem ser fatores determinantes para essas inadequações ${ }^{(28,29)}$. Estratégias de intervenção nessa realidade são urgentes e devem ser preocupação da família, da escola e do poder público.

Segundo Katon, Flores e Salmerón ${ }^{(30)}$, o perfil metabólico, incluindo as variáveis lipídicas, está fortemente relacionado ao desenvolvimento sexual. No entanto, o fato de todos os adolescentes do presente estudo encontrarem-se na fase púbere garantiu maior homogeneidade dos dados. As meninas apresentaram maiores valores nos níveis séricos de TG. Alguns trabalhos não encontraram diferenças entre os gêneros ${ }^{(31,32)}$, enquanto outros, coincidindo com os resultados observados, mostraram valores superiores para o gênero feminino ${ }^{(25,33-35)}$.

De acordo com as correlações apresentadas, verificou-se que a disponibilidade de lipídeos para consumo não esteve relacionada com o perfil lipídico dos adolescentes, a não ser em duas situações inesperadas nas quais o HDL se relacionou positivamente com a disponibilidade de AGS ( $\mathrm{r}=0,21$; $p<0,05)$ e o LDL se relacionou negativamente com a disponibilidade de AGP $\omega-6(\mathrm{r}=-0,25 ; p<0,05)$. Sabe-se que os AGS são potencialmente aterogênicos, estando relacionados ao aumento do CT e do LDL e não de HDL. O consumo desproporcional de AGP $\omega$ - 6 e $\omega$-3 também pode ter efeito aterogênico, aumentando os níveis de $\mathrm{LDL}^{(15)}$. Tais discrepâncias podem ser explicadas pelo fato da lista de compras da família poder inferir no consumo, mas não obrigatoriamente predizer o real consumo dos alimentos.

O presente estudo, assim como a $\mathrm{POF}^{(2)}$, não levou em consideração os alimentos adquiridos fora do ambiente domiciliar, componentes de lanches ou refeições prontas 
realizadas em outras localidades. Provavelmente, esse fato corrobora a justificativa da dificuldade em relacionar os dados apresentados. Supõe-se que, ao não se considerar tais alimentos, o consumo poderia ser subestimado, tornando a disponibilidade ineficiente em predizer alterações nos lipídeos sanguíneos.

Provavelmente, um número maior de listas, a inclusão dos produtos adquiridos fora do âmbito familiar pelo adolescente e a associação da lista com algum inquérito que leve em conta o consumo alimentar seriam recursos úteis na complementação dos dados. Outro ponto importante é a divergência de informações existentes entre tabelas e rótulos, que podem sub ou superestimar a composição lipídica dos mesmos.

O CT demonstrou associação com a disponibilidade de lipídeos, sendo que os adolescentes com mais de $35 \%$ de lipídeos para consumo apresentaram mais chances de alterações. Em outro estudo com adolescentes, Guedes et al ${ }^{(24)}$ verificaram que, ao analisar a ingestão de lipídeos totais, as meninas com excesso demonstraram chance 1,93 (IC95\% 1,66-2,17) maior de ter CT elevado. Os meninos apresentaram 1,61 (IC95\% 1,42-1,83) mais chances. Neste caso, verifica-se que, apesar da dificuldade em relacionar os lipídeos disponíveis para consumo com os lipídeos séricos, a lista de compras foi capaz de apresentar uma associação importante.

\section{Referências bibliográficas}

1. World Health Organization. Nutrition in adolescence - issues and challenges for the health sector. Issues in adolescent health and development. Geneva: WHO; 2005.

2. Levy-Costa RB, Sichieri R, Pontes NS, Monteiro CA. Household food availability in Brazil: distribution and trends (1974-2003). Rev Saude Publica 2005;39:530-40.

3. Brasil. Instituto Brasileiro de Geografia e Estatística. Pesquisa de orçamentos familiares - POF 2008-2009: despesas, rendimentos e condições de vida. Rio de Janeiro: IBGE; 2010.

4. Campos W, Stabelini Neto A, Bozza R, Ulbrich AZ, Bertin RL, Mascarenhas LP et al. Physical activity, lipid consumption and risk factors for atheroscleorosis in adolescents. Arq Bras Cardiol 2010;94:601-7.

5. Santos TM. Lipídios. In: Dutra-de-Oliveira JE, Marchini JS, editors. Ciências nutricionais. 2 ed. São Paulo: Sarvier; 2008. p.107-21.

6. Sposito AC, Caramelli B, Fonseca FA, Bertolami MC, Afiune Neto A, Souza $A D$, et al; Sociedade Brasileira de Cardiologia. IV Brazilian Guideline for Dyslipidemia and Atherosclerosis prevention: Department of Atherosclerosis of Brazilian Society of Cardiology. Arq Bras Cardiol 2007;88 (Suppl 1):2-19.

7. Brasil. Instituto Nacional de Estudos e Pesquisas Educacionais Anísio Teixeira [homepage on the Internet]. Censo Escolar [cited 2008 Apr 21]. Available from: http://portal.inep.gov.br/basica-censo-escolar-matricula

8. Chaves OC. Associação dos determinantes do estado nutricional dos pais com o estado nutricional dos adolescentes em Viçosa-MG [tese de mestrado]. Viçosa: Universidade Federal de Viçosa; 2009.

9. Brasil. Ministério da Saúde - Datasus [homepage on the Internet]. Informações de saúde: Viçosa-2007 [cited 2008 Mar 03]. Available from: http://tabnet. datasus.gov.br/cgi/deftohtm.exe?ibge/cnv/popmg.def
A utilização da lista de compras pode ter sido uma limitação deste estudo, pois se sabe que, independentemente do método escolhido para quantificar a ingestão alimentar e/ou disponibilidade, é difícil obter dados válidos e confiáveis, visto não haver um método considerado padrão-ouro. A dificuldade em relacionar a disponibilidade de lipídeos para consumo com o perfil lipídico sérico de adolescentes pode ser explicada, em parte, pelos erros inerentes ao processo de avaliação dietética. No entanto, a lista de compras permite conhecer a disponibilidade de alimentos no contexto no qual o indivíduo está inserido, sendo esta uma condição imprescindível na identificação dos hábitos alimentares, planejamento eficaz de intervenção e orientação nutricional.

Em conclusão, este estudo sugere que a maior disponibilidade de lipídeos para consumo nos domicílios é um fator que pode estar relacionado a níveis de colesterol total mais elevado em adolescentes. Visto que a adolescência constitui um período crítico para a incorporação de hábitos alimentares e para instalação de alterações no estado nutricional, é de fundamental importância programas específicos de atenção à saúde do adolescente, voltados para educação e aconselhamento individual e familiar, os quais poderiam prevenir ou minimizar problemas em curto e longo prazo.

10. De Faria ER. Critérios diagnósticos e fatores de risco para síndrome metabólica, em adolescentes que já apresentaram a menarca, de escolas públicas de Viçosa-MG [tese de mestrado]. Viçosa Universidade Federal de Viçosa; 2007.

11. Tanner JM. Growth at adolescence. Oxford: Blackwell Scientific; 1962.

12. Barbosa KB, Rosado LE, Franceschini SC, Priore SE. Instrumentos de inquérito dietético utilizados na avaliação do consumo alimentar em adolescentes: comparação entre métodos. ALAN 2007;57:43-50.

13. Franco G. Tabela de composição química dos alimentos. 9 ed. São Paulo: Atheneu; 2008.

14. Institute of Medicine. Dietary reference intakes for energy, carbohydrate, fiber, fatty acids, cholesterol, protein and amino acids (Macronutrients). Food and Nutrition Board. Washington: National Academy Press; 2002.

15. Sociedade Brasileira de Hipertensão, Sociedade Brasileira de Cardiologia, Sociedade Brasileira de Endocrinologia e Metabologia, Sociedade Brasileira de Diabetes, Associação Brasileira para Estudos da Obesidade. I Diretriz de prevenção da aterosclerose na infância e na adolescência. Arq Bras Cardiol 2005;85 (Suppl 1):3-28.

16. Philippi ST, Latterza AR, Cruz AT, Ribeiro LC. Adapted food pyramid: a guide for a right food choice. Rev Nutr 1999;12:65-80.

17. Friedwald WT, Levy RI, Fredrickson DS. Estimation of the concentration of low-density lipoprotein cholesterol in plasma, without use of the preparative ultracentrifuge. Clin Chem 1972;18:499-502.

18. Toral N, Slater B, Silva MV. Food consumption and overweight in adolescents from Piracicaba, São Paulo, Brazil. Rev Nutr 2007;20:449-59. 
19. Bertin RL, Karkle EN, Ulbrich AZ, Stabelini Neto A, Bozza R, Araújo IQ et al. The nutritional status and dietary intake of adolescents in public schools in the city of São Mateus do Sul, in the state of Paraná, Brazil. Rev Bras Saude Matern Infant 2008;8:435-43.

20. Toselli S, Argnani L, Canducci E, Ricci E, Gualdi-Russo E. Food habits and nutritional status of adolescents in Emilia-Romagna, Italy. Nutr Hosp 2010;25:613-21.

21. Barbosa KB, Volp AC, Renhe IR, Stringheta PC. Omega-3 and 6 fatty acids and implications on human health. Rev Soc Bras Alim Nutr 2007;32:129-45.

22. Carvalho DF, Paiva AA, Melo AS, Ramos AT, Medeiros JS, Medeiros CC et al. Blood lipid levels and nutritional status of adolescents. Rev Bras Epidemiol 2007;10:491-8.

23. Mendes GA, Martinez TL, Izar MC, Amancio OM, Novo NF, Matheus SC et al. Lipid profile and nutrition counseling effects in adolescents with family history of premature coronary artery disease. Arq Bras Cardiol 2006;86:361-5.

24. Guedes DP, Guedes JE, Barbosa DS, Oliveira JA, Stanganelli LC. Cardiovascular risk factors in adolescents: biological and behavioral indicators. Arq Bras Cardiol 2006;86:439-50.

25. Moreira C, Santos R, Vale S, Santos PC, Abreu S, Marques Al et al. Ability of different measures of adiposity to identify high metabolic risk in adolescents. J Obes 2011;2011:578106.

26. Pereira PB, Arruda IK, Cavalcanti AM, Diniz AS. Lipid profile of schoolchildren from Recife, PE. Arq Bras Cardiol 2010;95:606-13.

27. Rentfro AR, Nino JC, Pones RM, Innis-Whitehouse W, Barroso CS, Rahbar $\mathrm{MH}$ et al. Adiposity, biological markers of disease, and insulin resistance in Mexican American adolescents, 2004-2005. Prev Chronic Dis 2011;8:A40.

28. Zhang L, Qin LQ, Liu AP, Wang PY. Prevalence of risk factors for cardiovascular disease and their associations with diet and physical activity in suburban Beijing, China. J Epidemiol 2010;20:237-43.

29. Beck CC, Lopes AS, Giuliano IC, Borgatto AF. Cardiovascular risk factors in adolescents from a town in the Brazilian South: prevalence and association with sociodemographic variables. Rev Bras Epidemiol 2011;14:36-49.

30. Katon JG, Flores YN, Salmerón J. Sexual maturation and metabolic profile among adolescents and children of the Health Worker Cohort Study in Mexico. Salud Publica Mex 2009;51:219-26.

31. Faria ER, Franceschini SC, Pelúzio MC, Priore SE. Estado nutricional e dislipidemias de acordo com o sexo, em adolescentes atendidos em um programa específico de Viçosa - MG. Rev Bras Nutr Clin 2005;21:83-8.

32. Ribas SA, Silva LC. Dyslipidemia in schoolchildren from private schools in Belém. Arq Bras Cardiol 2009;92:446-51.

33. Brotons Cuixart C, Gabriel Sánchez R, Muñiz García J, Ribera Solé A, Málaga Guerrero S, Sáenz Aranzubia PE et al. Pattern of the distribution of total cholesterol and cHDL cholesterol Spanish children and adolescents: Ricardin Study. Med Clin (Barc) 2000;115:644-9.

34. Franca E, Alves JG. Dysplidemia among adolescents and children from Pernambuco. Arq Bras Cardiol 2006;87:722-7.

35. Lunardi CC, Petroski EL. Body mass index, waist circumference and skinfolds for predicting lipid abnormalities in 11 years old children. Arq Bras Endocrinol Metab 2008;52:1009-14. 